\title{
Observation of the transition to an insulating state consistent with a Wigner solid in a high-density $2 \mathrm{D}$ hole gas
}

\author{
P.J. Rodgers ${ }^{a}$, C.J.G.M. Langeraka, B.L. Gallagher ${ }^{a}$, R.J. Barraclough ${ }^{a}$, M. Henini ${ }^{a}$, \\ T.J. Foster ${ }^{\mathrm{a}}$, G. Hill' ${ }^{\mathrm{b}}$, S.A.J. Wiegers ${ }^{\mathrm{c}}$ and J.A.A.J. Perenboom ${ }^{\mathrm{c}}$ \\ "Department of Physics, University of Nottingham, UK \\ ${ }^{\mathrm{D}}$ Department of Electronic Engineering, University of Sheffield, UK \\ ${ }^{\mathrm{c}}$ High Field Magnet Laboratory and Research Institute for Materials, University of Nijmegen, The Netherlands
}

\begin{abstract}
We have observed a high-field transition into a high-resistance state at filling factor $\nu$ just below $2 / 7$ in a high-density, high-mobility two-dimensional hole gas (2DHG). The state shows clear threshold behaviour consistent with a strongly pinned Wigner solid. The transition is at lower filling factor than the value of $\sim 0.37$ recently reported by Santos et al. [7] for a low-density $2 \mathrm{DHG}$, and higher than the value of $\sim 0.22$ usually found in equivalent $2 \mathrm{D}$ electron systems. Our experiment shows no indication of a re-entrant behaviour of the insulating phase. We believe that this may be the highest-carrier-density Wigner-transition observed.
\end{abstract}

At sufficiently low temperatures and low carrier densities a two-dimensional conductor is expected to undergo a transition into an insulating Wigner solid, WS. At zero magnetic field this should occur at a critical value of the ratio $r_{\mathrm{s}}=a / a_{\mathrm{B}} \approx 37$ [1], where $a$ is the mean interelectron distance and $a_{\mathrm{B}}$ is the Bohr radius. One thus expects the critical carrier density to be proportional to the effective mass, $m^{*}$. In a magnetic field the carrier's zero point motion is confined to cyclotron orbits. In the high field limit $a / l_{\mathrm{c}}$ becomes the parameter of importance (where the magnetic length $l_{\mathrm{c}}=\sqrt{\hbar / e B}$ ) which is independent of $m^{*}$. The Wigner transition now occurs at a critical Landau level filling factor $\nu_{\mathrm{c}}$. Theory yields a high field value of $\nu_{c} \sim 1 / 6.5$ [2]. For conductors which are close to the Wigner transition at zero field, application of a magnetic field will lead to the transition occurring at larger filling factors than this asymptotic value. The form of the critical filling factor $\nu_{c}\left(r_{s}\right)$ between zero and the high-field limit is as yet not accurately known $[3,4]$. This is partly because the

Correspondence to: B.L. Gallagher, Department of Physics, University of Nottingham, Nottingham NG7 2RD, UK. effective mass of the carriers also determines the importance of the inter Landau level, LL, mixing and the extent of the carrier wavefunction. For a given magnetic field the LL energy separation, $\hbar \omega_{\mathrm{c}}$, is inversely proportional to $m^{*}$. For large $m^{*}$ and moderate magnetic field it can be small compared to the Coulomb energy and the fractional quantum Hall (FQH) energy gap $\Delta$. In this situation $\Delta$ can be substantially reduced and the Wigner crystal can become the ground state. Defining an LL mixing parameter $\lambda$ as the ratio of $\Delta$ to the cyclotron energy, Yoshioka [5] estimates that close to $\nu=\frac{1}{3}$ the WS becomes the ground state when $\lambda \sim 6$.

The situation is further complicated by the fact that at specific filling factors the FQH state can be the ground state even when one is at values of $\nu$ below $\nu_{\mathrm{c}}$. Magnetotransport and optical experiments on 2D electrons in high-quality GaAs$(\mathrm{Ga}, \mathrm{Al}) \mathrm{As}$ heterostructures [6] indicate that the Wigner transition occurs for $\nu$ just above $\frac{1}{5}(\nu \sim$ 0.22). The FQH state still seems to be the ground state at $\frac{1}{5}$ and one thus sees the re-entrant behaviour around $\frac{1}{5}$. Very recently Santos et al. [7] have reported a possible Wigner transition in a high-quality low-density $\left(0.4 \times 10^{11} \mathrm{~cm}^{-2}\right) 2 \mathrm{D}$ 
hole gas with an effective mass of $\sim 0.3 m_{\mathrm{e}}$. The transition starts at the much higher value of $\nu \sim 0.37$ and is re-entrant around the $\frac{1}{3} \mathrm{FQH}$ state. Such samples have much larger values of $r_{\mathrm{s}}$ than the corresponding electron systems, furthermore the LL mixing parameter of $\sim 5$ is very large and is interpreted as the dominant factor leading to the large reduction in $\nu_{c}$.

In this paper we present measurements on a 2D hole gas of much higher density. These have values of $r_{\mathrm{s}}$ and $\lambda$ intermediate between those of typical 2D electron samples and those of Santos et al. [7]. Our results are consistent with the Wigner transition occurring at the intermediate filling factor of $\nu \sim 0.28$.

The Hall bar sample used was a GaAs( $\mathrm{Ga}, \mathrm{Al}) \mathrm{As}$ heterostructure, grown by $\mathrm{MBE}$ on the (311)A plane of a semi-insulating substrate. Sample NU703P is doped with $\mathrm{Si}$, which is incorporated as an acceptor on this plane. It has a hole carrier density of $n=1.4 \times 10^{11} \mathrm{~cm}^{-2}$ and a mobility of $\sim 200000 \mathrm{~cm}^{2} / \mathrm{V} \mathrm{s}$. The hole effective mass, determined from cyclotron resonance experiments up to $18 \mathrm{~T}$, is estimated to be $0.40 \mathrm{~m}_{\mathrm{e}}$ at $22 \mathrm{~T}$ [8]. Samples were contacted with $\mathrm{Au}(5 \mathrm{~nm})-\mathrm{Zn}(10 \mathrm{~nm})-\mathrm{Au}(200 \mathrm{~nm})$ evaporated contacts annealed at $\sim 500^{\circ} \mathrm{C}$ giving contact resistances of less than $30 \Omega$.

Transport measurements at low currents $(\sim 1 \mathrm{nA})$ and low frequencies $(<10 \mathrm{~Hz})$ were performed using isolated, battery-powered, bandpass pre-amplifiers and digital lock-in amplifiers. A hybrid magnet provided fields up to $25 \mathrm{~T}$. Temperatures down to $50 \mathrm{mK}$ were achieved by mounting the samples in the mixing chamber of a plastic tailed dilution refrigerator. It was possible, by pumping out the ${ }^{3} \mathrm{He}$ from the dilution refrigerator, to provide further cooling to $\sim 30 \mathrm{mK}$ for a period of a few minutes. Due to the small rapid field fluctuations in the Bitter magnet and the large vibrations induced by the cooling system for the Bitter magnet coils, it is possible that the $2 \mathrm{DHG}$ was not in thermal equilibrium with the mixing chamber; we estimate that the hole temperatures may be of order $20 \mathrm{mK}$ higher than the mixing chamber. Plastic packages were used to hold the samples minimising eddy current heating inherent with metallic packages.
Noise levels were reduced through the use of fine twisted pair connections into the cryostat. It was found necessary to further limit noise levels by placing small $(<300 \mathrm{pF})$ capacitors across each twisted pair. For low field values this had a negligible effect upon the measurements; however, when the sample enters the insulating phase the AC impedance of the capacitor $(\sim 60 \mathrm{M} \Omega)$ in parallel with the input impedance of the measurement system $(\sim 100 \mathrm{M} \Omega)$ provided a significant shunt to the measured sample resistance. The data presented here has been numerically corrected to allow for these effects.

In order to examine the pinning of the observed insulating state the AC differential resistance of the sample as a function of DC bias was measured at an excitation current of $1 \mathrm{nA}$. The DC bias was provided by mercury cells and a potential divider network. Care was taken to ensure that the net impedance of this circuit was large $(\sim 50 \mathrm{M} \Omega)$ to reduce the shunting effects described above.

In fig. 1 we show the magnetic field dependence of the longitudinal resistance $R_{x x}$ of the sample at $50 \mathrm{mK}$ up to $17 \mathrm{~T}$. This diagram demonstrates a good range of fractional states indicating the high quality of the material. The results are similar to those of Davies et al. [9]. In the inset diagram the longitudinal resistance at high magnetic fields (up to $24 \mathrm{~T}$ ) is plotted on a much larger scale. It shows the occurrence of an insulating state in the extreme quantum limit. Above $\nu=\frac{1}{3}$ a step in the resistance is observed and above $\nu=\frac{2}{7}(B=20.4 \mathrm{~T}) R_{x x}$ rises rapidly, reaching values larger than $1 \mathrm{M} \Omega / \square$ : this is about 100 times larger than any of the low-field features. A minimum at the $\frac{2}{7}$ state is not observed, for this sample, within the range of temperatures $(>50 \mathrm{mK})$ covered by this experiment.

The temperature dependence of $R_{x x}$ in the region of the insulating state is plotted in fig. 2 . A dramatic increase in $R_{x x}$ is observed with decreasing temperature. The change in $R_{x x}$ is measured in the range $19-25 \mathrm{~T}$, which includes the shoulder above $\nu=\frac{1}{3}$ and the field $(20.4 \mathrm{~T})$ where one would expect to observe the $\nu=\frac{2}{7}$ minimum. In contrast to this behaviour the maximum between $\nu=\frac{2}{5}$ and $\frac{1}{3}(15.5 \mathrm{~T})$ shows a very 


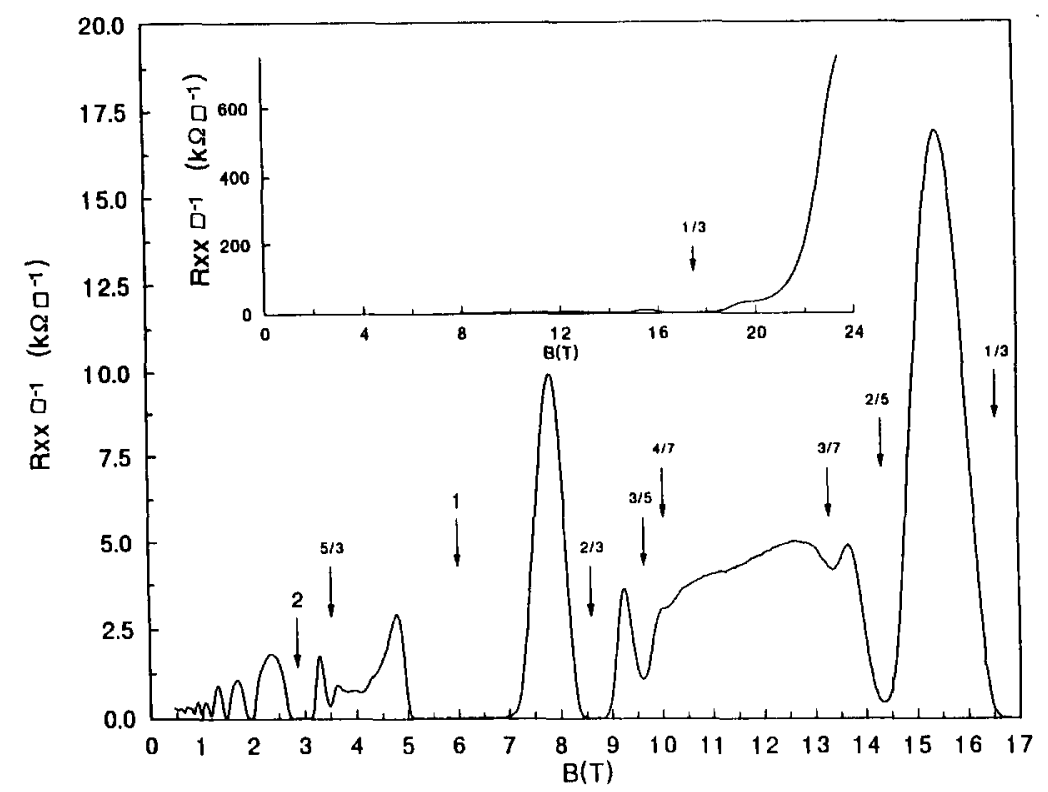

Fig. 1. The low-field longitudinal resistance for sample NU703; observed fractions are indicated by their filling factors. The inset shows the high-field insulating phase, on a much larger scale, appearing at magnetic fields above $20.4 \mathrm{~T}(\nu \sim 0.28)$.

weak temperature dependence down to $\sim 30 \mathrm{mK}$. These results contrast with those by Santos et al. [7] who demonstrated a re-entrant insulating phase around the $\frac{1}{3}$ fraction at temperatures up to $\sim 150 \mathrm{mK}$. For $T>100 \mathrm{mK}$ the observed temperature dependence is consistent with activated behaviour with a gap energy rising to $\sim 350 \mathrm{mK}$.

In fig. 3 the Hall resistance $R_{x y}$ is shown as a function of magnetic field at three different tem-

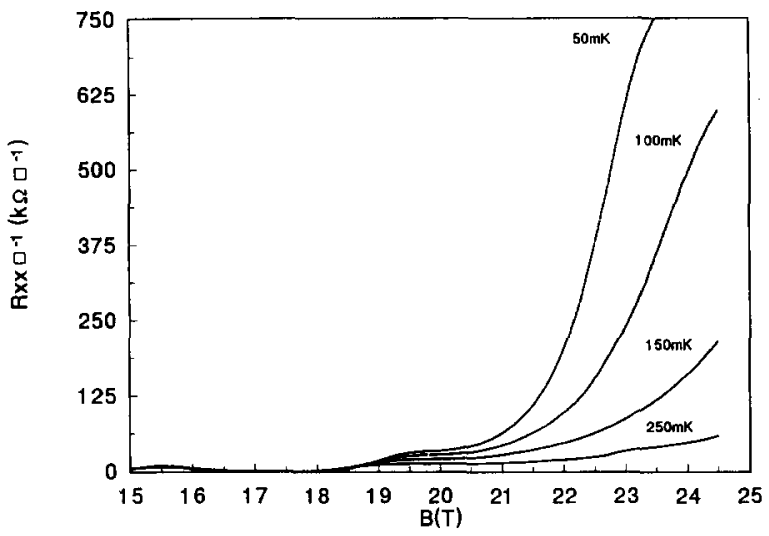

Fig. 2. Temperature dependence of the high-magnetic-field insulating transition in the longitudinal resistance, $R_{x x}$. peratures. The low-field trace $A(0-10 \mathrm{~T})$ is taken at $50 \mathrm{mK}$ and demonstrates good quantisation. The high-field (10-25 T) traces B, C and D are taken at $100 \mathrm{mK}, 150 \mathrm{mK}$ and $250 \mathrm{mK}$ respectively. They all display a quantised plateau corresponding to the $\frac{1}{3} \mathrm{FQHE}$ state. Strong divergence from the classical Hall curve is observed above $19 \mathrm{~T}$. The temperature dependence of the divergence is analogous to that observed in $R_{x x}$ (see fig. 2). By $250 \mathrm{mK}$ the divergence is small, $R_{x x}$ being almost exactly the classical Hall curve. Again no anomolous behaviour is observed below $\frac{1}{3}$ in contrast to Santos et al. [7].

The AC differential resistance $R_{x x}$ against DC bias voltage over the sample is shown in fig. 4 . It shows a family of curves taken at $50 \mathrm{mK}$ at a series of fixed magnetic fields where $R_{x x}$ shows insulating behaviour. At the field where one expects the $\frac{2}{7}$ FQHE state we see only a very small dependence of $R_{x x}$ upon bias to surprisingly large electric fields. This may be due to the rather flat dispersion relation of the holes which requires that one go much further in $k$-space to cause a given heating of the carriers. As one moves beyond the $\frac{2}{7}$ into the insulating state a strong nonlinearity develops and a clear plateau 


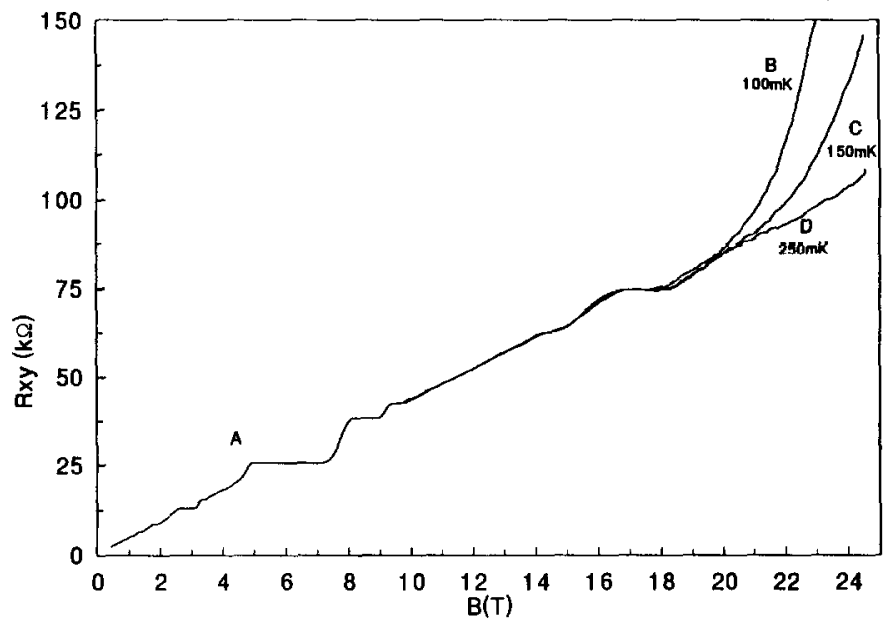

Fig. 3. The low-field $(0-10 \mathrm{~T})$ Hall resistance, $R_{x y}$, and the temperature dependence at high magnetic fields (10-24 T), including those fields at which an insulating state is seen in $R_{x x}$.

is observed before the onset of breakdown of the high resistance state $(\sim 70 \mathrm{mV}, \sim 70 \mathrm{~V} / \mathrm{m})$. The breakdown threshold fields are very close to those found in high-quality $2 \mathrm{D}$ electron systems by Williams et al. [10] but seem to be much larger than those of Santos et al. [7].

Our $R_{x x}, R_{x y}$ and threshold results are very similar to those taken as evidence for a WS around $\frac{1}{5}$ in the 2DEG and around $\frac{1}{3}$ in the

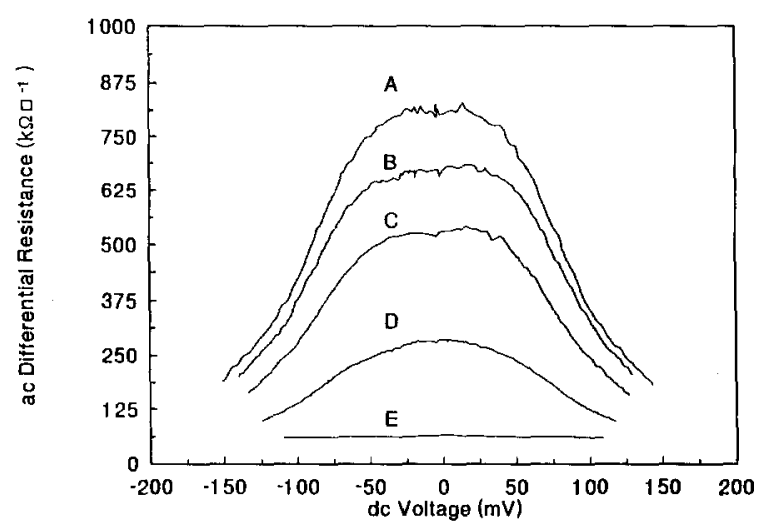

Fig. 4. The AC differential resistance at various filling factors, displaying the insulating behaviour, versus $\mathrm{DC}$ voltage bias. Traces $\mathrm{A}, \mathrm{B}, \mathrm{C}, \mathrm{D}$ and $\mathrm{E}$ are taken at filling factors $\nu=0.253,0.255,0.258,0.267$ and $0.293\left(\sim \frac{2}{7}\right)$ with corresponding magnetic fields of $23.0,22.9,22.6,21.8$ and $19.9 \mathrm{~T}$ respectively. low-density $2 \mathrm{DHG}$ and are consistent with a critical filling factor $\nu_{\mathrm{c}} \approx 0.28$. Transport measurement alone cannot uniquely associate the insulating state with the Wigner solid. We see essentially identical behaviour for different contacts and different sections of the same device so it is clearly a bulk effect characteristic of the 2DHG. It is however still possible that the observed transition is due to magnetic freeze-out. One argument against this is that one expects this to occur when the magnetic field has significantly reduced the extent of the carrier wavefunction. For the $2 \mathrm{DEG}$ the magnetic length becomes smaller than $a_{\mathrm{B}}$ at $\sim 6 \mathrm{~T}$ and in high-quality samples freeze-out occurs at values large compared to this. The corresponding field for our sample is $\sim 220 \mathrm{~T}$ which is very much larger than the field at which we observe the transition.

The hole-dispersion relationships show strong nonparabolicity and anisotropy leading to $m^{*}$ being dependent on $B$ and the Fermi energy. The cyclotron hole mass has been determined to high accuracy in our material [8]. At $B=4.67 \mathrm{~T}$, which is close to the insulating transition of Santos et al., $m^{*}=0.304 m_{\mathrm{e}}$ in agreement with their value [7]. By 18.2 T however this has risen to $0.389 m \mathrm{~m}$ and extrapolation to $B \approx 22 \mathrm{~T}$ gives $m^{*} \approx 0.40 m_{\mathrm{e}}$. For $n=1.4 \times 10^{11} \mathrm{~cm}^{-2}$ we have 
$r_{\mathrm{s}} \approx 9$ which is a little lower than the value of $\sim 12$ for the low-density hole gas and considerably higher than the typical value of $\sim 2$ for the 2DEG. Similarly the values of $\lambda$ for our sample close to the observed transition is $\sim 3$, compared to $\sim 5$ for the Santos et al. [7] sample and $\sim 1$ for the electron gases. It thus seems highly plausible that a Wigner transition should occur in our sample at a value intermediate between those observed in these other systems.

In conclusion we have observed a high-field transition into an insulating state at filling factor $\nu$ just below $\frac{2}{7}$ in a high-density, high-mobility two-dimensional hole gas (2DHG). The state shows well-defined threshold behaviour consistent with a strongly pinned Wigner solid. No indication of a re-entrant behaviour of the insulating phase is observed.

\section{Acknowledgements}

We would like to acknowledge C.J. Mellor, J.M. Chamberlain, P.C. Main and L. Eaves for useful discussions, and the staff of the Nijmegen
High Magnetic Field Laboratory for their technical assistance. This work was financially supported by the SERC (UK) and the FOM (the Netherlands).

\section{References}

[1] B. Tanatar and D.M. Ceperely, Phys. Rev. B 39 (1989) 5005.

[2] P.K. Lam and S.M. Girvin, Phys. Rev. B 30 (1984) 473.

[3] P.M. Platzman, in: The Physics of the Two-Dimensional Electron Gas, NATO Adv. St. Inst. Ser. B. Vol. 157, eds. J.T. Devreese and F.M. Peeters (Plenum Press, New York, 1987) p. 97.

[4] S.T. Chui and K. Esfarjani, Europhys. Lett. 14 (4) (1991) 361.

[5] D. Yoshioka, J. Phys. Soc. Jpn. 55 (1986) 885.

[6] See the extensive references of refs. [7] and [10].

[7] M.B. Santos, Y.W. Suen, M. Shayegan, Y.P. Li, L.W. Engel and D.C. Tsui, Phys. Rev. Lett. 68 (1992) 1188.

[8] S.D. Hawksworth, J.M. Chamberlain, M. Henini, J. Singleton, T.J.B.M. Janssen, G. Hill and S. Summers, to be published.

[9] A.G. Davies, R. Newbury, M. Pepper, J.E.F. Frost and G.A.C. Jones, Phys. Rev. B 44 (1991) 13128.

[10] F.I.B. Williams et al., Phys. Rev. Lett. 66 (1991) 3285. 